\title{
Prédiction de l'insulino-résistance par les ratios de lipoprotéines chez les adultes béninois
}

\author{
Charles SOSSA ${ }^{1,2^{*}}$, Victoire AGUEH ${ }^{1}$, Jacques SAIZONOU ${ }^{1}$, \\ Paraïso Noel MOUSSILIOU ${ }^{1}$, Alphonse KPOZEHOUEN ${ }^{1}$, \\ Michel MAKOUTODE ${ }^{1}$ et Hélène DELISLE ${ }^{2}$ \\ ${ }^{1}$ Institut Régional de Santé Publique (IRSP) de Ouidah, BP 384 Ouidah, Bénin ; Tél: (+229) 21341674 ou \\ 21341675 , Fax: (+229) 21341672. \\ ${ }^{2}$ TRANSNUT, Centre Collaborateur OMS sur la Transition Nutritionnelle et le Développement, Département \\ de Nutrition, Faculté of Médecine, Université de Montréal. CP 6128 succ. Centre-ville, Montréal Qc, Canada, \\ H3C $3 J 7$; Tél: $1514343-6111$ poste 25219. \\ *Auteur correspondant, E-mail : Sossajero@yahoo.com
}

\section{RESUME}

Des controverses subsistent sur force prédictive de l'insulino-résistance par les ratios de lipoprotéines chez les Afro-américains. L'objectif est de déterminer la force prédictive de l'insulino-résistance (IR) par les ratios triglycérides-par-HDL-cholestérol (TG/HDL-C) et cholestérol total-par-HDL-cholestérol (CT/HDL-C) chez des adultes béninois. L'étude est de type transversal, issue d'une enquête longitudinale sur le risque cardiométabolique et a inclut 416 sujets âgés de 29 à 69 ans. Les mesures anthropométriques et les dosages de la glycémie à jeun, de l'insulinémie, du CT, du HDL-C et des TG sont réalisés. L'IR est définie par le $75^{\text {ème }}$ percentile des valeurs de «l'indice d'évaluation de modèle d'homéostasie pour l'insulino-résistance» (HOMA IR). Les valeurs prédictives de l'IR par les ratios TG/HDL-C et CT/HDL-C sont déterminées par les aires sous les courbes (AUC) de la «fonction d'efficacité du récepteur» (ROC). Les concentrations plasmatiques moyennes des lipides ont été en général plus élevées chez les IR que chez les non IR sauf pour les triglycérides : $0,9 \pm 0,5 \mathrm{mmol} / \mathrm{L}$ versus $0,8 \pm 0,4 \mathrm{mmol} / \mathrm{L}(\mathrm{p}=0,138)$ chez les femmes, le cholestérol total : 4,7 $\pm 1,7 \mathrm{mmol} / \mathrm{L}$ versus $4,3 \pm 1,2 \mathrm{mmol} / \mathrm{L}(\mathrm{p}=0,161)$ et le HDL-Cholestérol : $1,2 \pm 0,5 \mathrm{mmol} / \mathrm{L}$ versus $1,34 \pm 0,5$ $\mathrm{mmol} / \mathrm{L}(\mathrm{p}=0,105)$ chez les hommes. Les AUC ROC de prédiction de l'IR par le TG/HDL-C sont inférieures à 0,70 chez les femmes et les hommes, les obèses et les non obèses. L'AUC ROC de prédiction de l'IR par le CT/HDL-C est supérieure à 0,70 seulement chez les femmes : 0,71 (0,64-0,78). Le ratio TG/HDL-C n'est pas un prédicteur de l'IR dans la population d'étude alors que le ratio CT/HDL-C prédit l'IR chez les femmes seulement. Ces résultats contribueront au dépistage précoce de l'IR chez les femmes aux fins de la mise en place de mesures préventives.

(C) 2015 International Formulae Group. All rights reserved.

Mots clés : Prédiction, insulino-résistance, lipoprotéines, dyslipidémies, Bénin.

\section{INTRODUCTION}

La résistance à l'insuline (IR) caractérisée par une réponse physiologique insuffisante de l'insuline est un facteur de risque pour les maladies cardiovasculaires (CVD) et le diabète de type 2 (Bonora et al., 
2007 ; Luke et al., 2012). Plus de 346 millions de personnes à travers le monde souffrent de diabète, et en 2030 leur nombre sera plus que doublé si des mesures préventives ne sont pas prises (WHO, 2011). Lorsqu'une anomalie du métabolisme du glucose est détectée par une glycémie élevée, une importante proportion de cellules- $\beta$ des ilots de Langerhans est peutêtre déjà physiologiquement défectueuse (Ferrannini et Mari, 2004). Les interventions sur le style de vie y compris l'alimentation et l'activité physique sont susceptibles d'améliorer la sensibilité à l'insuline lorsque l'IR est dépistée précocement et que le métabolisme du glucose n'est pas encore perturbé (Colberg et Grieco, 2009).

La technique éprouvée du clamp hyperinsulinémique-euglycémique utilisée pour le diagnostic de l'IR est complexe et peu pratique pour les besoins cliniques de dépistage de routine. Des indices simples de l'IR comme "l'indice d'évaluation de modèle d'homéostasie pour l'insulino-résistance» (HOMA IR) ont été proposés (Bonora et al., 2000), mais l'absence de standardisation de la technique du dosage de l'insuline limite l'utilisation du HOMA IR. Le ratio triglycérides par lipoprotéines à haute densité de cholestérol (TG/HDL-C) a été identifié comme un indice clinique simple de l'IR chez les Caucasiens (Li et al., 2008 ; Kim-Dorner et al., 2010 ; Chiang et al., 2011).

Des controverses subsistent quant à la force prédictive de cet indice chez les Afroaméricains (Sumner et al., 2005a ; Sumner et al., 2005b ; Sumner et al., 2010a). Des auteurs (Kim-Dorner et al., 2010) ont observé dans une étude transversale de 50 Caucasiens et 99 Afro-américains, que les aires sous les courbes (AUC) de la « fonction d'efficacité du récepteur» (ROC) de TG/HDL-C pour la prédiction de l'IR, selon le HOMA-IR, étaient de 0,77 et 0,64 , respectivement. Les auteurs avaient conclu que la relation entre TG/HDL$\mathrm{C}$ et la résistance à l'insuline était ethnospécifique et n'était pas un indice prédictif de l'IR chez les Afro-Américains. D'autres auteurs (Sumner et al., 2010a) ont rapporté que le ratio TG/HDL-C prédit l'IR chez les hommes et non chez les femmes afroAméricaines. Dans une étude multi-ethnique (Li et al., 2008) menée aux Etats-Unis, l'aire sous la courbe ROC du ratio TG/HDL-C pour prédire l'hyper-insulinémie était de 0,77 pour les Blancs non-hispaniques, 0,75 chez les Noirs non hispaniques et de 0,74 chez les Américains d'origine mexicaine. Les auteurs concluent que le TG/HDL-C peut être un indicateur clinique pour l'hyper-insulinémie chez les adultes non diabétiques indépendamment de l'ethnie et de la race. Des valeurs seuils ethno-spécifiques de TG/HDLC ont été proposées pour l'IR : 3,0 pour les Blancs non-hispaniques et Américains d'origine mexicaine, 2,0 pour les Noirs non hispaniques (Li et al., 2008), et de 2,5 pour les hommes afro-américains (Sumner et al., 2010a).

Chez les Africains sub-sahariens, les études réalisées sur la valeur prédictive de l'IR par le ratio TG/HDL-C (Knight et al., 2012) ont rapporté que l'AUC ROC pour la prédiction de l'IR par le TG/HDL-C est audessous du seuil de 0,70 chez les femmes noires sud-africaines et ghanéennes en surpoids, en comparaison aux Caucasiennes chez qui l'AUC ROC était de 0,76. Les études menées aux Seychelles (Bovet et al., 2006) ont montré que l'AUC ROC pour la prédiction de l'IR par le TG/HDL-C était de 0,65 chez les non diabétiques d'origine africaine en surpoids. La force prédictive du TG/HDL-C chez les hommes est peu documentée chez les Africains sub-sahariens. De même, la capacité prédictive de l'IR par le ratio CT/HDL-C n'est pas connue dans ce groupe ethno-racial. L'objectif de cette étude était d'apprécier la force prédictive de l'IR par les ratios TG/HDL-C et CT/HDL-C chez les adultes du Bénin.

\section{MATERIEL ET METHODES \\ Cadre d'étude}

L'étude s'est déroulée au Sud du Bénin dans la grande métropole urbaine (Cotonou), en milieu semi-urbain (ville de Ouidah) situé à 
40 kilomètres de Cotonou et dans sa périphérie rurale.

\section{Population d'étude}

La population d'étude est constituée d'adultes béninois âgés de 29 à 69 ans.

\section{Méthodes d'étude Type d'étude}

Il s'agit d'une étude transversale issue d'une étude longitudinale et observationnelle sur l'évolution sur une période de quatre ans des facteurs de risque cardiométabolique (RCM) chez des adultes béninois (juin 2006 à octobre 2010). La présente étude exploite les données du dernier suivi de l'étude longitudinale.

\section{Taille de l'échantillon}

Pour estimer le nombre minimal de sujets nécessaires à l'utilisation de la statistique de l'aire sous la courbe ROC, l'IR a été utilisée comme variable dépendante principale. En fixant les erreurs de première espèce $\alpha$ et de deuxième espèce $\beta$ à $5 \%$ et $20 \%$ respectivement, l'aire sous la courbe ROC attendue à 0,65 , la valeur de l'aire sous la courbe ROC dans l'hypothèse nulle à 0,50 et le ratio cas négatifs / cas positifs à 4 , la taille minimale d'échantillon nécessaire est de 185 sujets (37 cas positifs et 148 cas négatifs) en utilisant le logiciel «MedCalc version 13.1.2, Ostend, Belgium ». Un effectif de 416 sujets composé de 208 femmes et 208 hommes ont participé à la présente étude.

\section{Technique d'échantillonnage}

Les 416 sujets de la présente étude proviennent des participants de l'étude longitudinale observationnelle initiale qui a inclus des sujets apparemment en bonne santé âgés de 25-60 ans sélectionnés aléatoirement par échantillonnage par grappes dans la plus grande ville de Cotonou, la petite ville de Ouidah, et la périphérie rurale de Ouidah au Bénin. Parmi les 541 sujets initialement inclus dans l'étude longitudinale (200 en milieu urbain, 171 en milieu semi-urbain et 170 en milieu rural), un effectif de 416 ont été retrouvés au dernier suivi.

\section{Procédure de collecte des données}

Les mesures anthropométriques ont été prises par des enquêteurs formés. Des échantillons de sang veineux ont été prélevés le matin à jeun et conservés dans la glace puis centrifugés dans les deux heures subséquentes. Les échantillons ont été stockés à $-30{ }^{\circ} \mathrm{C}$ jusqu'à l'analyse dans le Laboratoire de Biochimie de l'Institut des Sciences Biomédicales Appliquées de Cotonou.

Les variables de l'étude

* La variable dépendante l'insulino résisistance

L'indice HOMA-IR obtenu par la formule (insulinémie $\mathrm{x}$ glycémie) / 22,5 (Wallace et al., 2004) a été utilisée comme méthode indirecte d'appréciation de l'IR. L'insulinémie à jeun a été dosée au Laboratoire de Biochimie de l'Institut des Sciences Biomédicales Appliquées de Cotonou à l'aide de la technique ELISA (Enzyme-Linked Immunosorbent Assay) avec des kits IBL basés sur le principe du sandwich (IBL International, 2011). La sensibilité analytique du test était de $1,76 \mu \mathrm{IU} / \mathrm{ml}$, les coefficients de variation inter-dosages et intradosages variaient de 2,88 à $5,99 \%$ et 1,79 à $2,6 \%$ respectivement. Le dosage était exempt d'une sensibilité croisée à la pro-insuline. Le $75^{\text {ème }}$ percentile des valeurs de HOMA-IR a été considéré comme la valeur seuil de l'IR, tel que proposé par d'autres études (Wallace et al., 2004). La glycémie a été dosée par la méthode de glucose oxydase (Wu, 2005) dans le plasma des sujets à jeun.

* La variable indépendante : les ratios TG/HDL-C et CT/HDL-C

Les lipides ont été dosés chez les sujets à jeun au Laboratoire de Biochimie de l'Institut des Sciences Biomédicales Appliquées de Cotonou par la méthode enzymatique colorimétrique (Wu, 2005) en utilisant les kits de Elitech group (Sées, France). 
* Les variables de contrôle : le sexe et l'obésité abdominale de contrôle.

Le sexe et l'obésité sont les variables

\section{Analyse statistique des données}

Les données sont analysées avec le logiciel SPSS, version 20.0 (SPSS Inc., Chicago, IL). Les AUC ROC de TG/HDL-C et de CT/HDL-C pour prédire l'IR ont servi à apprécier les valeurs prédictives. L'aire minimale sous la courbe ROC pour une valeur prédictive acceptable est fixée à 0,70 (Hosmer et Lemeshow, 2000). La moyenne et l'écart type ont été calculés pour les variables quantitatives dont la distribution était normale. Les moyennes ont été comparées par le test $t$ de Student. Le niveau de signification statistique pour les tests est fixé à p inférieur à 0,05 .

\section{Considérations éthiques}

L'étude a été approuvée par les comités d'éthique de la Faculté de Médecine de l'Université de Montréal (Canada) et du Ministère de la Santé du Bénin. Le consentement éclairé écrit a été obtenu de chaque participant avant leur recrutement dans l'étude longitudinale.

\section{RÉSULTATS}

Caractéristiques sociodémographiques des sujets

Parmi les 416 sujets, 50\% sont des femmes. La moyenne d'âge des hommes est $37,2 \pm 10,1$ ans et celle des femmes est 38,9 \pm 9,9 ans. Les sujets résident en milieu rural $(31 \%)$, semi-urbain $(37,3 \%)$ et urbain $(31,7 \%)$.

Prévalence des facteurs de risque cardiométabolique selon l'insulinorésistance

Les valeurs de HOMA IR ne suivent pas une distribution normale et il a été procédé à une transformation logarithmique. Suivant la définition de l'IR à partir de
HOMA IR (HOMA>3.2 dans notre échantillon), 106 sujets (69 femmes et 37 hommes y compris quatre sujets diabétiques sous traitement antidiabétique) sont classés IR sur les 416, soit 25,9\% (Tableau 1). Les valeurs moyennes des lipides sanguins et de tour de taille sont, en général, plus élevées chez les IR en comparaison aux non IR. Chez les femmes, les concentrations moyennes en triglycérides ne diffèrent pas significativement selon l'IR ( $p=0,138)$. Il en est de même pour le cholestérol total $(\mathrm{p}=0,161)$ et le HDL-C ( $\mathrm{p}$ $=0,105)$ chez les hommes.

Prédiction de l'insulino-résistance par le TG/HDL-C le CT/HDL-C

Selon le sexe, la Figure 1 montre que l'aire sous la courbe ROC du ratio CT/HDL chez les femmes est supérieure à 0,70 contrairement à celle de CT/HDL chez les hommes. Les AUC ROC de TG/HDL-C sont inférieures à 0,70 chez les hommes et les femmes.

Selon le statut de tour de taille, la Figure 2 montre que les aires sous les courbes ROC de CT/HDL-C et TG/HDL-C sont inférieures à 0,70 tant chez les sujets qui présentent une obésité abdominale que chez les non obèses.

Valeur seuil prédictive de l'insulinorésistance par le CT/HDL-C chez les femmes

A partir de l'indice de Youden (sensibilité + spécificité - 1), la valeur seuil optimale du ratio $\mathrm{CT} / \mathrm{HDL}-\mathrm{C}$ prédictive de l'IR a été déterminée. Cette valeur seuil est de 3,5 correspondant à une sensibilité de 0,66 et une spécificité de 0,69 (Figure 1). Par ailleurs la valeur seuil de $\mathrm{CT} / \mathrm{HDL}-\mathrm{C} \geq 4,5 \mathrm{chez}$ les femmes correspond à une sensibilité de 0,43 et une spécificité de 0,80 . 

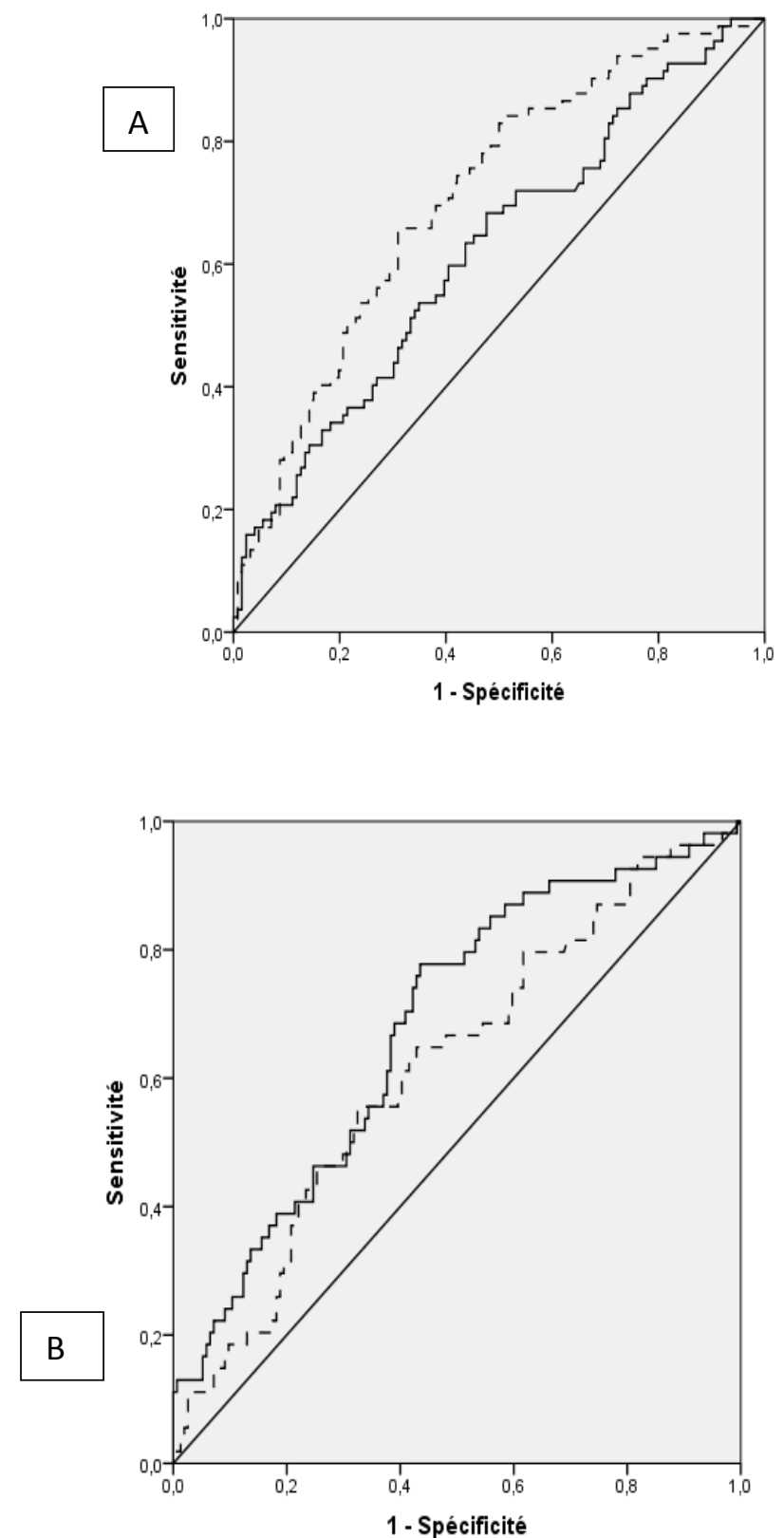

AUC CT/HDL-C : 0,71 (IC 95\% : 0,64-0,78). AUC TG/HDL-C : 0,62 (IC 95\% : 0,54-0,70).

AUC CT/HDL-C : 0,62 (IC 95\% : 0,53-0,70) AUC TG/HDL-C : 0,68 (IC 95\% : 0,60-0,76)

AUC : aire sous la courbe «ReceiverOpérating Charatéristics »; CT/HDL-C : ratio cholestérol total-par-HDL-cholestérol ; TG/HDL-C : ratio triglycérides-par-HDL-cholestérol ; Légende: - - - CT/HDL-C TG/HDL-C

Figure 1 : Comparaison des aires sous les courbes ROC prédictives de l'insulino-résistance par les ratios TG/HDL-C et T/HDL-C isolé chez les femmes (A) et les hommes (B). 

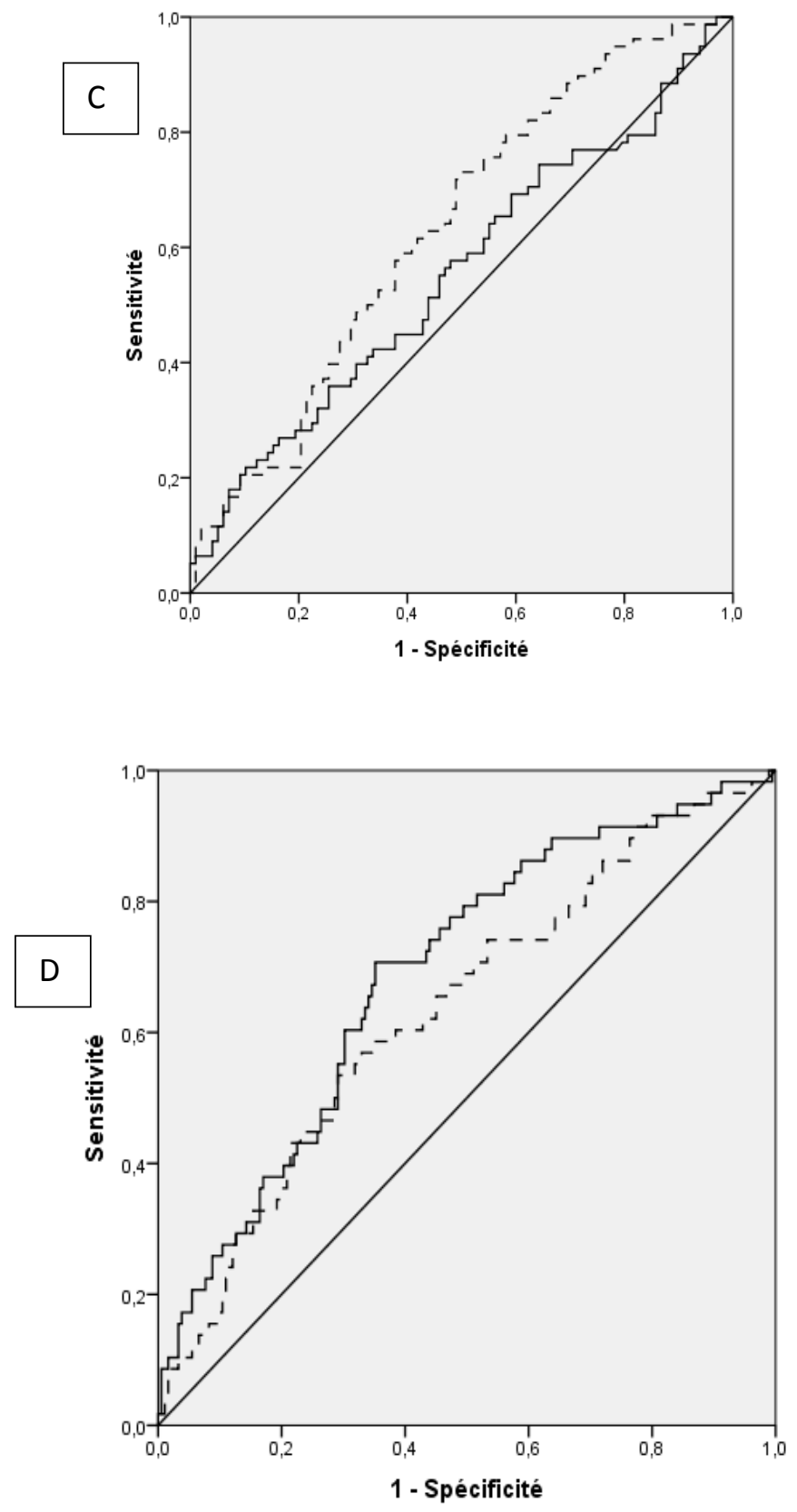

AUC CT/HDL-C : 0,63 (IC 95\% : 0,55-0,71).

AUC TG/HDL-C : 0,55 (IC 95\% : 0,47-0,64).

AUC CT/HDL-C : 0,64 (IC 95\% : 0,55-0,72). AUC TG/HDL-C : 0,69 (IC 95\% : 0,61-0,76).

AUC : aire sous la courbe « Receiver Opérating Charatéristics »; CT/HDL-C : ratio cholestérol total-par-HDLcholestérol ; TG/HDL-C : ratio triglycérides-par-HDL-cholestérol ; Légende: - - - CT/HDL-C TG/HDL-C

Figure 2 : Comparaison des aires sous les courbes ROC prédictives de l'insulino-résistance par les ratios TG/HDL-C et T/HDL-C isolé chez les obèses $(C)$ et les non obèses $(D)$. 
Tableau 1 : Facteurs de risque cardiométabolique selon l'insulino-résistance.

\begin{tabular}{|c|c|c|c|c|c|c|}
\hline & & Femmes & & & Hommes & \\
\hline Facteurs de risque & IR $(\mathrm{n}=39)$ & Non IR(n=139) & $\mathbf{p}$ & IR $(\mathrm{n}=37)$ & Non IR(n=171) & $\mathbf{p}$ \\
\hline Tour de taille & $88,9 \pm 12,6$ & $83,4 \pm 12,5$ & $\mathbf{0 , 0 0 3}$ & $89,2 \pm 12,1$ & $81,2 \pm 10,2$ & $<0,001$ \\
\hline Cholestérol total & $4,7 \pm 1,4$ & $4,5 \pm 1,2$ & 0,216 & $4,7 \pm 1,7$ & $4,3 \pm 1,2$ & 0,161 \\
\hline HDL-C & $1,2 \pm 0,4$ & $1,4 \pm 0,4$ & $<0,001$ & $1,2 \pm 0,5$ & $1,34 \pm 0,5$ & 0,105 \\
\hline CT/HDL-C & $4,2 \pm 1,6$ & $3,3 \pm 1,3$ & $<0,001$ & $4,2 \pm 1,8$ & $3,6 \pm 1,5$ & $\mathbf{0 , 0 1 2}$ \\
\hline Triglycérides & $0,9 \pm 0,5$ & $0,8 \pm 0,4$ & 0,138 & $1,4 \pm 1,0$ & $0,9 \pm 0,5$ & $<0,001$ \\
\hline TG/HDL-C & $1,0 \pm 1,0$ & $0,7 \pm 0,5$ & $<0,001$ & $1,3 \pm 1,0$ & $0,8 \pm 0,6$ & $<0,001$ \\
\hline
\end{tabular}

R: Insulini-résistants ;

Non IR : Non insulino-résistants

HDL-C: HDL cholestérol

CT/HDL-C: ratio cholestérol total-par-HDL-cholestérol

TG/HDL-C:ratio triglycérides-par-HDL-cholestérol

Les valeurs sont exprimées en moyenne \pm écart type

p-value pour le test $t$ de Student pour échantillons indépendants 


\section{DISCUSSION}

La présente étude a examiné la force prédictive de l'IR par les ratios TG/HDL-C et CT/HDL-C chez les adultes béninois (Africains subsahariens). Les résultats montrent que le ratio TG/HDL-C n'est pas un prédicteur de l'IR dans la population d'étude alors que le ratio CT/HDL-C prédit l'IR chez les femmes avec une valeur seuil optimale de 3,5 .

\section{Le TG/HDL-C n'est pas un prédicteur de l'insulino-résistance dans la population d'étude}

Dans des études sur la relation entre l'IR et les lipides, des auteurs avaient plutôt observé qu'à l'inverse des Caucasiens, le taux de triglycérides ainsi que le ratio TG/HDL-C n'étaient pas prédictifs de l'IR mesurée par le HOMA IR chez les Afro-américains, à partir de l'analyse de l'AUC ROC de prédiction de l'IR (Kim-Dorner et al., 2010). Les résultats de la présente étude concordent aussi avec ceux d'autres études menées en 2005 (Sumner et al., 2005a). La faiblesse, voire l'absence de lien entre l'IR et les ratios TG/HDL-C a été aussi rapportée dans une étude multi-ethnique auprès de 1904 Américains (Karnchanasorn et al., 2012). Ces auteurs ont trouvé que parmi les sujets présentant le syndrome métabolique (SMet) qui est la traduction clinique de l'IR (Reaven, 2011), 32\% des Caucasiens nonHispaniques et $28 \%$ des non-Hispaniques noirs n'étaient pas dans le tercile supérieur du HOMA IR. De même, parmi les sujets IR, figuraient $25 \%$ de Caucasiens nonHispaniques, et $36 \%$ de non-Hispaniques noirs qui n'avaient pas de SMet. En outre, dans une étude multi-ethnique portant sur des femmes noires Ouest-Africaines, SudAfricaines et Afro-américaines, il a été rapporté d'après les courbes ROC, que le ratio TG/HDL-C n'était pas prédictif de l'IR dans ces populations. En particulier, l'AUC ROC chez les femmes Ouest-africaines était la plus faible $(0,63 \pm 0,07)$ (Knight et al., 2012). Des auteurs avaient déjà souligné la nécessité de réaliser des études prospectives afin de déterminer si une reformulation ethnospécifique des seuils pour les dyslipidémies du SMet pourrait optimiser la relation entre l'IR et les composantes de SMet chez les Noirs (Sumner et al., 2010b).

L'activité élevée de la lipoprotéine lipase chez les Noirs par rapport aux Caucasiens a été évoquée pour expliquer ces observations (Buthelezi et al., 2000 ; Despres et al., 2000 ; Reaven and McLaughlin, 2006). Il est tout de même nécessaire d'approfondir la relation entre les lipides et l'IR en utilisant par exemple le rapport apoB / apoA-I, qui reflèterait mieux l'équilibre des taux de cholestérol athérogénique et antiathérogénique (Sniderman et Faraj, 2007; Walldius et Jungner, 2007). L'IR est plus fréquente chez les Noirs que les Caucasiens chez qui la relation entre l'IR et les dyslipidémies est plus forte comme le témoigne la valeur prédictive élevée de l'IR par le ratio TG/HDL-C (Gonzalez-Chavez et al., 2011). L'IR est plus fréquente chez les Afro-Américains par rapport aux Caucasiens mais les Afro-Américains ont des concentrations sériques de triglycérides plus faibles (Sumner et al., 2005a; Sumner et al., 2005b). Ainsi, si l'IR et les concentrations en triglycérides ne sont pas étroitement liées, il est donc peu probable que la concentration plasmatique de triglycérides ou le ratio TG/HDL-C soit utiles dans l'identification des individus IR. L'IR n'expliquerait que $16 \%$ de la variabilité des triglycérides selon les mêmes auteurs (Sumner et al., 2005b).

\section{Le CT/HDL-C est un prédicteur de l'insulino-résistance chez les femmes}

Des études antérieures ont montré, dans le même sens que les résultats de la présente étude, que dans une population à risque de maladie cardiovasculaire, le ratio CT/HDL-C identifiait mieux les personnes à risque que le score de Framingham (Bersot et al., 2003). En effet, les deux-tiers environ de cholestérol plasmatique se trouvent dans le «Low density lipoprotein cholestérol» (LDL), et par conséquent, le cholestérol total et le LDL-C sont étroitement liés (Pintó et Ros, 2000). En effet, le cholestérol total, inclut aussi les lipoprotéines riches en triglycérides nonobstant que l'hypertriglycéridémie soit 
rare chez les Africains subsahariens (Sodjinou et al., 2008; Sumner et al., 2010b). Le ratio CT/HDL-C a donc l'avantage d'exprimer plus convenablement la proportion entre les lipides athérogènes et ceux anti-athérogènes. De même, le HDL-C/CT, qui indique la proportion de taux de cholestérol protecteur par rapport au reste non protecteur (Pintó et Ros, 2000), tient compte du LDL-C qui a été le premier indice de risque de MCV identifié et la principale cible pour la thérapie (Genest et al., 2009).

Valeur seuil de CT/HDL-C prédictive de l'insulino-résistance chez les femmes

La valeur seuil prédictive de l'IR par le CT/HDL-C chez les femmes est de 3,5 et correspond à une sensibilité de 0,66 et une spécificité de 0,69. Cette valeur seuil est inférieure à celle rapportée par d'autres auteurs : CT/HDL-C $\geq 4,5$ (Millan et al., 2009) qui correspond dans notre échantillon, à une sensibilité de 0,43 et une spécificité de 0,80. La détermination de la valeur seuil optimale, devrait prendre en considération le fait que l'état d'IR n'implique pas un traitement aux effets secondaires potentiellement graves, il n'est donc pas indispensable de rechercher une spécificité élevée. À l'inverse, au vue des complications graves de l'IR qui peuvent être évitées si le diagnostic et les mesures préventives sont précoces, la sensibilité du test diagnostic est donc plus utile (Delacour et al., 2005).

Les limites de la présente étude tiennent à l'absence de valeurs seuils universelles et spécifiques pour le HOMA-IR (Ybarra et al., 2005; Alvarez et al., 2006), bien qu'il est fortement corrélé $(r=0,82)$ avec les valeurs $\mathrm{du}$ verrouillage euglycémique hyper-insulinémique (Bonora et al., 2000). Par ailleurs, il n'a pas été pris en compte dans les analyses, l'effet modulateur des facteurs tels que l'alimentation et l'activité physique sur les lipides sanguins. En fin, l'étude a été effectuée uniquement dans la région sud du Bénin.

\section{Conclusion}

La présente étude a montré que le ratio TG/HDL-C n'est pas un prédicteur de l'IR dans la population d'étude alors que le CT/HDL-C peut être utilisé comme un prédicteur de l'IR chez les femmes. Ces résultats contribueront, en milieu clinique, à l'optimisation du dépistage précoce des sujets IR chez les femmes en vue de la mise en place des mesures préventives.

\section{REMERCIEMENTS}

Les auteurs expriment leur gratitude aux initiateurs de l'étude longitudinale, aux enquêteurs de terrain et les participants à l'étude. L'étude a été financée par l'Agence Canadienne de Développement International (ACDI).

\section{REFERENCES}

Alvarez MM, Vieira AC, Moura AS, da Veiga GV. 2006. Insulin resistance in Brazilian adolescent girls: association with overweight and metabolic disorders. Diabetes Research and Clinical Practice, 74(2): 183-188.

Bersot TP, Pepin GM, Mahley RW. 2003. Risk determination of dyslipidemia in populations characterized by low levels of high-density lipoprotein cholesterol. American Heart Journal, 146(6): 10521059.

Bonora E, Kiechl S, Willeit J, Oberhollenzer F, Egger G, Meigs JB, Bonadonna RC, Muggeo M. 2007. Insulin resistance as estimated by homeostasis model assessment predicts incident symptomatic cardiovascular disease in caucasian subjects from the general population: the Bruneck study. Diabetes Care, 30(2): 318-324.

Bonora E, Targher G, Alberiche M, Bonadonna RC, Saggiani F Zenere MB, Monauni T, Muggeo M. 2000. Homeostasis model assessment closely mirrors the glucose clamp technique in the assessment of insulin sensitivity: studies in subjects with various degrees of glucose tolerance and insulin sensitivity. Diabetes Care, 23(1): 57-63. 
Bovet P, Faeh D, Gabriel A, Tappy L. 2006. The prediction of insulin resistance with serum triglyceride and high-density lipoprotein cholesterol levels in an East African population. Archives of Internal Medicine, 166(11): 1236-1237.

Buthelezi EP, van der Merwe MT, Lonnroth PN, Gray IP, Crowther NJ. 2000. Ethnic differences in the responsiveness of adipocyte lipolytic activity to insulin. Obesity Research, 8(2): 171-178.

Chiang JK, Lai NS, Chang JK.Koo M. 2011.Predicting insulin resistance using the triglyceride-to-high-density lipoprotein cholesterol ratio in Taiwanese adults. Cardiovasc. Diabetol., 10: 93.

Colberg SR, Grieco CR. 2009. Exercise in the treatment and prevention of diabetes. Curr. Sports Med. Rep., 8(4):169-175.

Delacour H, Servonnet A, Perrot A, Vigezzi J, Ramirez J. 2005. La courbe ROC (receiver operating characteristic): principes et principales applications en biologie clinique. An. Bio. Clin., 63(2): 145-154.

Despres JP, Couillard C, Gagnon J, Bergeron J, Leon AS, Rao DC, Skinner JS, Wilmore JH, Bouchard C.2000. Race, visceral adipose tissue, plasma lipids, and lipoprotein lipase activity in men and women: the Health, Risk Factors, Exercise Training, and Genetics (HERITAGE) family study. Arteriosclerosis, Thrombosis, and Vascular Biology, 20(8): 1932-1938.

Ferrannini E, Mari A. 2004. Beta-Cell function in type 2 diabetes. Metabolism: Clinical and Experimental, 63(10): 12171227.

Genest J, McPherson R, Frohlich J, Anderson T, Campbell N. Carpentier A, Couture P, Dufour R, Fodor G,Francis GA, Grover S, Gupta M, Hegele RA, Lau DC, Leiter L, Lewis GF, Lonn E, Mancini GB, Pearson GJ, Sniderman A, Stone JA, Ur E. 2009. 2009 Canadian Cardiovascular Society/Canadian guidelines for the diagnosis and treatment of dyslipidemia and prevention of cardiovascular disease in the adult - 2009 recommendations.
Canadian Journal of Cardiology, 25(10): 567-579.

Gonzalez-Chavez A, Simental-Mendia LE, Elizondo-Argueta S. 2011. Elevated triglycerides/HDL-cholesterol ratio associated with insulin resistance. Cirugia y Cirujanos, 79(2):126-131.

Hosmer D, Lemeshow S. 2000. Applied Logistic Regression (2nd edn). John Wiley \& Sons Inc: New York.

IBL International. 2011. Insulin ELISA: Instruction for Use. IBL International: Hamburg.

Karnchanasorn R, Ou HY, Chuang LM, Chiu KC. 2012. Insulin resistance is not necessarily an essential element of metabolic syndrome. Endocrine.

Kim-Dorner S-J, Deuster PA, Zeno SA, Remaley AT,Poth M. 2010. Should triglycerides and the triglycerides to highdensity lipoprotein cholesterol ratio be used as surrogates for insulin resistance? Metabolism: Clinical and Experimental, 59(2): 299-304.

Knight MG, Goedecke JH, Ricks M, Evans J, Levitt NS, Tulloch-Reid MK, Sumner AE. 2012. The TG/HDL-C ratio does not predict insulin resistance in overweight women of African descent: a study of South African, African American and West African women. Ethnicity and Disease, 21(4): 490-494.

Li C, Ford ES, Meng YX, Mokdad AH, Reaven GM. 2008. Does the association of the triglyceride to highdensity lipoprotein cholesterol ratio with fasting serum insulin differ by race/ethnicity? Cardiovasc Diabetol., 7:19.

Luke JN, Brown AD, Brazionis L, O'Dea K, Best JD, McDermott RA, Wang Z, Rowley KG. 2013. Exploring clinical predictors of cardiovascular disease in a central australian aboriginal cohort. Eur J. Prev Cardiolog., 20(2): 246-253.

Millan J, Pinto X, Munoz A, Zuniga M, Rubies-Prat J, Pallardo LF, Massana L, Mangas A, Hernandez-Mijares A, Gonzalez-Santos P, Ascaso JF, PedroBotet J. 2009. Lipoprotein ratios: 
Physiological significance and clinical usefulness in cardiovascular prevention. Vasc. Health. Risk Manag., 5(7): 757765.

Pintó X, Ros E. 2000. Lípidosséricos y prediccióndelriesgo cardiovascular: importancia de loscocientescolesterol total/colesterol HDL y colesterol LDL/colesterol HDL. Clin. Invest. Arterioscl., 12: 267-284.

Reaven MG. McLaughlin T. 2006. Why the plasma TG/HDL-C concentration ratio does not predict insulin resistance in African Americans. Archives of Internal Medicine, 166(2): 249-250.

Reaven GM. 2011. Relationships among insulin resistance, type 2 diabetes, essential hypertension, and cardiovascular disease: similarities and differences. $J$. Clin. Hypertens, 13(4): 238-243.

Sniderman AD, Faraj M. 2007. Apolipoprotein B, apolipoprotein A-I, insulin resistance and the metabolic syndrome. Current Opinion in Lipidology, 18(6): 633-637.

Sodjinou R, Agueh V, Fayomi B,Delisle H. 2008. Obesity and cardio-metabolic risk factors in urban adults of Benin: relationship with socio-economic status, urbanisation, and lifestyle patterns. $B M C$ Public Health, 8(4): 84-97.

Sumner AE, Finley KB, Genovese DJ, Criqui MH, Boston RC. 2005a. Fasting triglyceride and the triglyceride-HDL cholesterol ratio are not markers of insulin resistance in African Americans. Archives of Internal Medicine, 165(12): 1395-1400.

Sumner AE, Vega GL, Genovese DJ, Finley KB, Bergman RN, Boston RC. 2005b. Normal triglyceride levels despite insulin resistance in African Americans: role of lipoprotein lipase. Metabolism: Clinical and Experimental, 54(7): 902-909.

Sumner AE, Harman JL, Buxbaum SG, Miller BV, Tambay AV. 2010a. The triglyceride/high-density lipoprotein cholesterol ratio fails to predict insulin resistance in African-American women: an analysis of Jackson Heart Study. Metab. Syndr. Relat. Disord., 8(6): 511514.

Sumner AE, Zhou J, Doumatey A, Imoisili OE, Amoah A,AcheampongJ, Oli J, Johnson T, Adebamowo C, Rotimi CN. 2010b. Low HDL-Cholesterol with Normal Triglyceride Levels is the Most Common Lipid Pattern in West Africans and African Americans with Metabolic Syndrome: Implications for Cardiovascular Disease Prevention. CVD. Prev. Control., 5(3): 75-80.

Wallace TM, Levy JC, Matthews DR. 2004. Use and abuse of HOMA modeling. Diabetes Care, 27(6): 1487-1495.

Walldius G, Jungner I. 2007. Is there a better marker of cardiovascular risk than LDL cholesterol? Apolipoproteins B and A-I new risk factors and targets for therapy. Nutrition, Metabolism and Cardiovascular Diseases, 17(8): 565-571.

World Health Organization. 2011. Diabetes. Fact sheet $\mathrm{N}^{\circ} 312$.Disponible sur http://www.who.int/mediacentre/factsheet s/fs312/en/. Consulté le 29 septembre 2011.

Wu AHB. 2005. Tielz Clinical Guide to Laboratory Tests (4 ${ }^{\text {th }}$ edn). Saunders: Philadelphia.

Ybarra J, Sanchez-Hernandez J, Pérez A. 2005. Non-HDL-cholesterol, waist circumference and the HOMA index: Correlations in the healthy. Prevention and Control., 1(2): 183-184. 